\title{
TerraSAR-X Calibration Results
}

Marco Schwerdt, Benjamin Bräutigam, Markus Bachmann, Björn Döring

Microwave and Radar Institute of the German Aerospace Center (DLR)

P.O.-Box 1116, D-82230 Weßling, Germany

Marco.Schwerdt@dlr.de

\begin{abstract}
TerraSAR-X is a satellite mission for scientific and commercial applications operating a highly flexible X-band SAR instrument with a multitude of different operation modes. As product quality is of crucial importance, the success or failure of the mission depends essentially on the method of calibrating TerraSAR-X in an efficient way during commissioning the entire system in a restricted time. Only then, product quality and the correct operation of the SAR system can be ensured. The paper describes the method of calibrating TerraSAR-X and final results derived from all calibration procedures.
\end{abstract}

\section{Scope and Strategy}

On June 15th, 2007, the first German SAR satellite for commercial and scientific applications, TerraSAR-X, was launched. The essential task of calibrating TerraSAR-X is to estimate and correct systematic error contributions throughout the complete SAR system and to tie-down image information (magnitude and phase) to reference units in geophysical terms. In addition to the high degree of flexibility of TerraSAR-X with different operation modes (StripMap, Spotlight, ScanSAR, right/left looking, etc.) and a tight performance with an absolute radiometric accuracy better than $1 \mathrm{~dB}$, the main goal after launch of the satellite is to provide calibrated and verified SAR data products as soon as possible. Thus, a strategy for an efficient but robust calibration method was developed [1]. Applying a novel antenna model approach the in-orbit calibration method can be sub-divided into six major tasks performed during commissioning of TerraSAR-X. The successive baseline calibration procedures are:

1. Geometric Calibration, to assign the SAR data to the geographic location on the earth's surface.

2. Antenna Pointing Determination, to obtain a correct beam pointing of the antenna.

3. Antenna Model Verification, to ensure the provision of the antenna patterns of all operation modes and the gain offset between different beams.

4. Relative Radiometric Calibration, for radiometric correction of SAR data within an illuminated scene.

5. Absolute Radiometric Calibration, for measuring the SAR system against standard targets with well known radar cross section (RCS).

However, the success of performing all these activities within a tight schedule for commissioning TerraSAR-X is essentially dependent on the stability of the instrument.
Thus, an accurate facility for the Internal Calibration (6th major task) was established and implemented for monitoring and compensating drift effects of the instrument.

\section{Internal Calibration}

Temperature drifts and internal hardware characteristics influence the radar signal path causing gain and phase fluctuation during data acquisition. For monitoring and compensating these effects the radar instrument of TerraSAR-X hosts an internal calibration facility comprising both conventional internal calibration pulses and the so called PNgating method, a further novel calibration method [2].

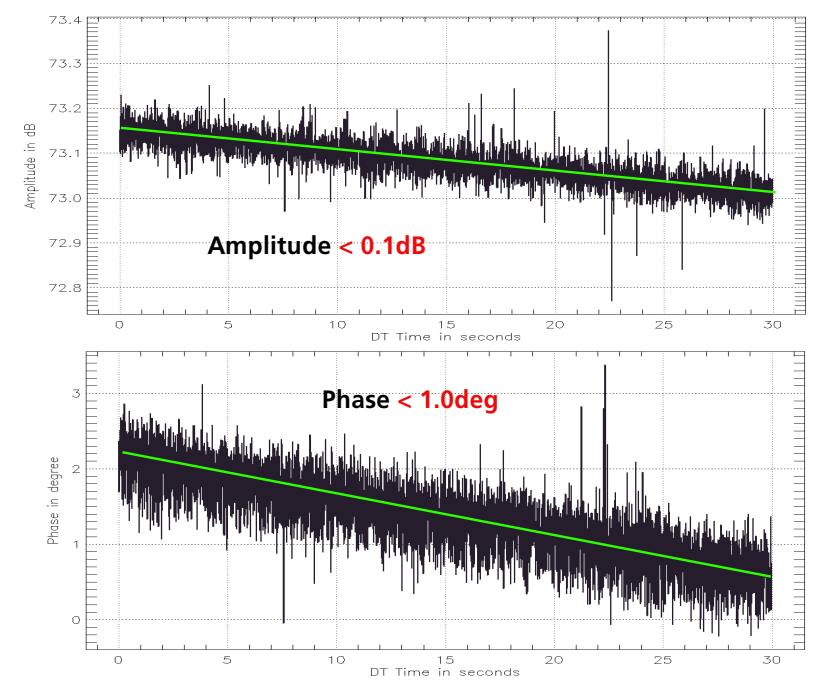

Figure 1: Residual drift error of the instrument by applying the internal calibration facility, black lines: radar pulses wihtin a datatake, green line: fit derived from calibration pulses. 
The amplitude and the phase drift of the TerraSAR-X instrument within a data take are shown in Fig. 1, whereby the black lines are the radar pulses and the green line is a fit derived from calibration pulses. Thus, by monitoring the instrument with internal calibration pulses even small drift effects can be compensated for down to an accuracy < $0.1 \mathrm{~dB}$ for the amplitude and better than $1 \mathrm{deg}$ for the phase. Furthermore, the novel PN-Gating method, developed and established for characterizing individual transmit/receive $(T / R)$ modules in operation of an active phased array antenna, has now been successfully verified for the first time in orbit by TerraSAR-X [3]. By this method the actual amplitude setting of each T/R module can be measured down to an accuracy of $0.2 \mathrm{~dB}$ and better than $2 \mathrm{deg}$ for the phase setting . Based on this accurate internal calibration facility all calibration procedures described in section 1 could be performed within the tight schedule of commissioning TerraSAR-X.

\section{Geometric Calibration}

The purpose of geometric calibration is the geometric assignment of the SAR system to the earth's surface. Two major effects influence the correct localization of the product: the internal electronic delay of the instrument and range offset/propagation delay by hydrostatic effects.

Measuring the TerraSAR-X system against passive targets with no internal delay these effects could be determined and compensated for. But also the annotation of the source packets was checked against systematic azimuth or range line shifts respectively.

The residual slant range offset as function of look angle is shown in Fig. 2. Thus, for TerraSAR-X a pixel localization accuracy of $30 \mathrm{~cm}$ could be achieved, i.e. the connection between the SAR data and the earth surface could be calibrated down to an accuracy of almost one magnitude better than the product specification with $2 \mathrm{~m}$.

By this accurately calibrated TerraSAR-X system the impact of propagation effects on the range delay could be verified for the first time by a SAR system [4].

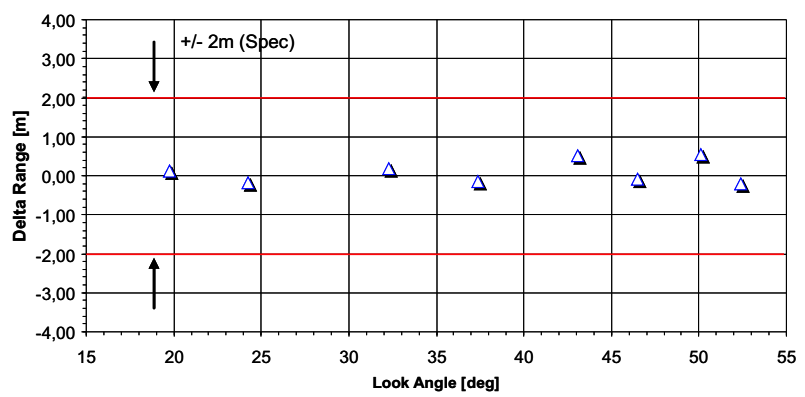

Figure 2: Residual slant range offset in dependence of the look angle after correcting for instrument delay and propagation effects.

\section{Antenna Pointing}

An important task is the determination of beam pointing errors coming from mechanical and electrical antenna misspointing as well as attitude control offsets. These errors are measured using an appropriate notch pattern over rain forest and by ground receivers. One example of measuring the beam pointing in elevation is shown in Fig. 3 by illuminating the Amazone rainforest with a notch pattern.

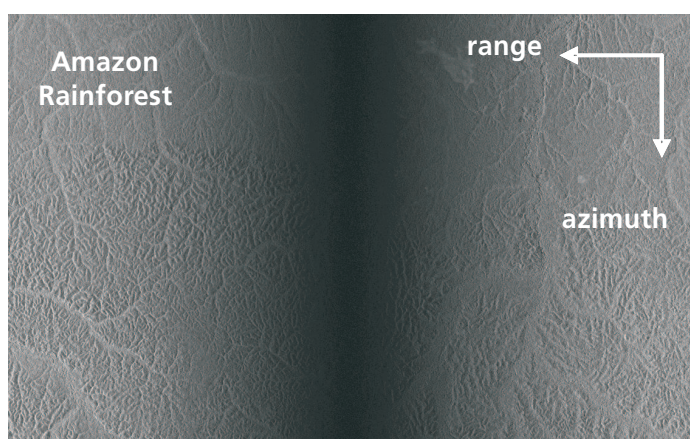

Figure 3: Amazon rainforest illuminated by a notch pattern in StripMap operation of TerraSAR-X.

As expected, the brightness in the mid of the scene is reduced due to the notch within the elevation pattern. Deriving the gamma profile of such data takes and applying the corresponding reference pattern derived from the antenna model, the offset of the notch and consequently the actual pointing of the TerraSAR-X antenna in elevation could be determined down to an accuracy better than the required $0.015 \mathrm{deg}$, as shown in Fig 4.

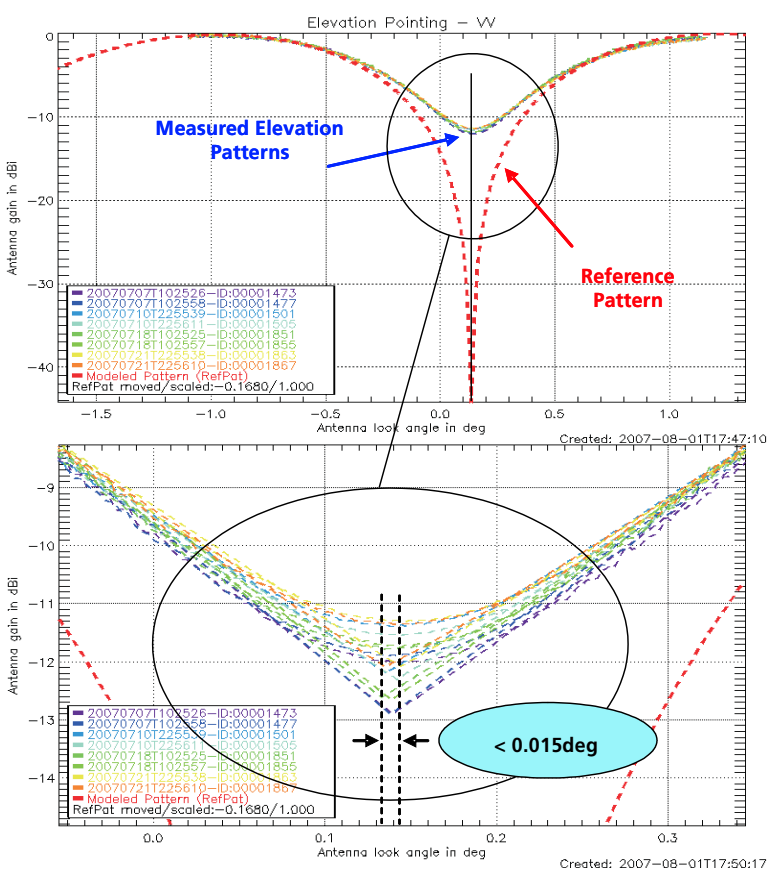

Figure 4: Beam pointing in elevation of TerraSAR-X determined by measured notch patterns across the rainforest. 
The beam pointing in flight direction could be determined likewise by notch patterns now operated in azimuth direction and measured by deployed ground receivers. The achieved pointing knowledge in azimuth is better than the required $0.002 \mathrm{deg}$ as well. Hence, by readjusting the attitude of the satellite the detected miss-pointing of the antenna beam could be removed and the remaining squint angle is in the order of the required pointing knowledge in both directions, i.e. negligible.

\section{Antenna Model Verification}

The characterization of the antenna is based on a precise antenna model. The verification of this antenna model was performed with a few selected beams really measured inflight and can be divided into three main tasks:

- measurements across the rainforest to verify the elevation pattern shape,

- ScanSAR measurements over rainforest to verify the calculated vs. simulated peak-to-peak gain offset between different beams and

- measurements using ground receivers to verify azimuth pattern shape.

The verification in flight shows that the maximum deviation between the antenna model and the measurements for both the shape within the main-beam and the gain-offset between different beams is less than $+/-0.2 \mathrm{~dB}$ (peak-topeak) [5].

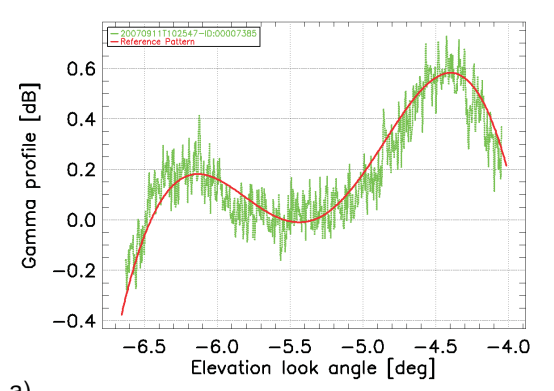

a)

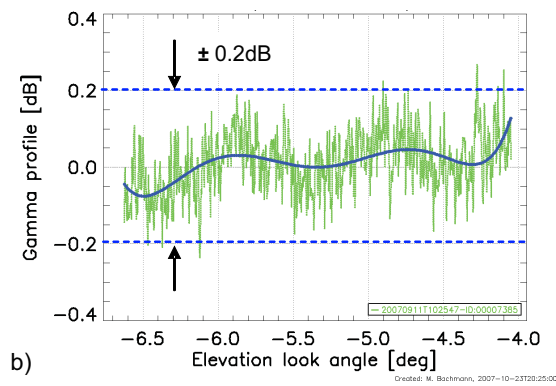

Figure 5: a) Comparison of the gamma profile (green) derived from a rain forest scene and the reference elevation pattern (red), b) difference between reference pattern and gamma profile, the blue line is a fit of this difference.
One example of verifying the shape of the elevation pattern is shown in Fig. 5. The slight noisy green curve in a) is the gamma profile derived by SAR data acquired over Amazon rainforest and the red line is the corresponding reference pattern (red line) derived by the antenna model. The deviation between both in Fig. 5b)and hence the accuracy of the antenna model is below $+/-0.2 \mathrm{~dB}$ (peak-to-peak).

By these excellent results [5] verifying a precise antenna model the effort for the relative and the absolute radiometric calibration could be reduced extremely because only a few selected beams had to be really measured in-flight.

\section{Radiometric Calibration}

The final step for radiometric calibration is the measurement of the whole TerraSAR-X system against reference targets with well known RCS. Analyzing the impulse response of these targets with respect to their RCS the absolute calibration factor can be derived from each target. One example of the absolute calibration factor derived from targets deployed within a swath is shown in Fig. 6. Separated according to the position of each target (near, mid and far position within the swath) and concentrating on the mean value of all measurements at one position, the variation within the swath is less than $+/-0.2 \mathrm{~dB}$. Consequently, the verification of the antenna model could be performed once again, now by a real end-to-end system test with deployed reference targets. The standard deviation of all measurements within one scene or across the whole swath defines the relative radiometric accuracy and for TerraSAR-X we have achieved $0.3 \mathrm{~dB}$ in StripMap operation.

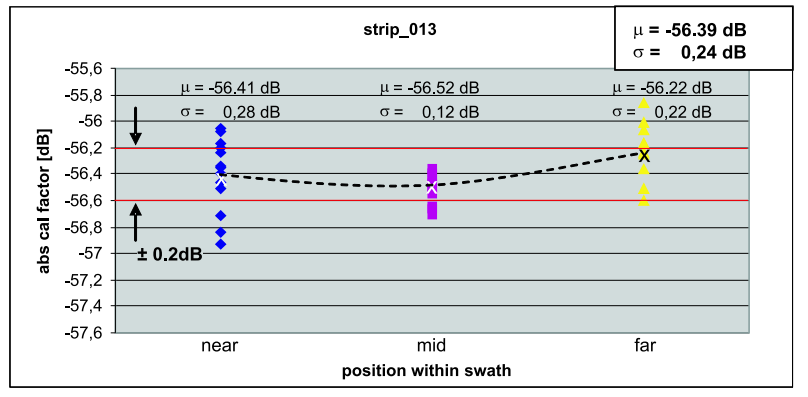

Figure 6: Absolute calibration factor derived from reference targets deployed across the swath in StripMap mode.

Separating the absolute calibration factor according to the beam ID, i.e. as function of look angle, the gain-offset between different beams could be likewise verified by deployed reference targets with an accuracy less than +/$0.2 \mathrm{~dB}$ [4]. Hence, absolute radiometric calibration of the whole TerraSAR-X system must be independent on both, the position of the targets deployed within the swath and the beam being operated. Because the shape of the antenna pattern and the gain-offset between different beams are already compensated by applying the antenna model during SAR data processing. And indeed, displaying the 
absolute calibration factor derived from all measurements only as function of measurement ID, as shown in Fig. 7, no significant dependency can be observed. Consequently, the absolute radiometric accuracy is defined by the standard deviation of all measurements. For TerraSAR-X we have achieved an absolute radiometric accuracy of $0.31 \mathrm{~dB}$ for StripMap operation during the CP of TerraSAR-X.

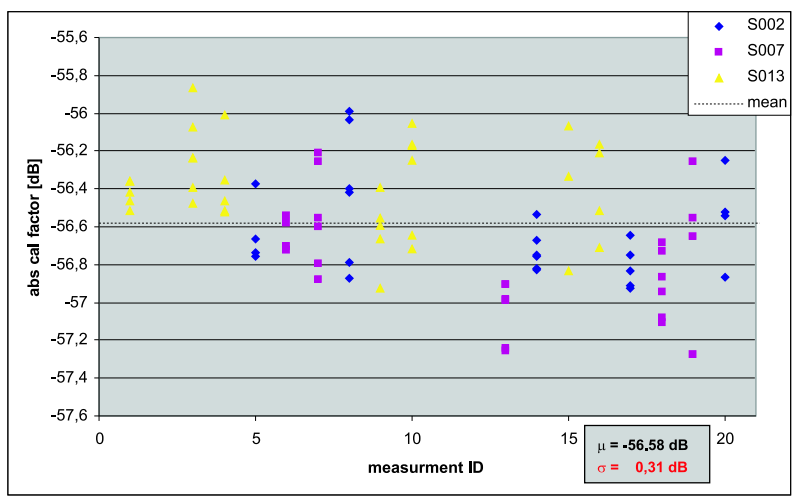

Figure 7: Absolute calibration factor versus measurement ID derived from all reference targets deployed and all StripMap beams being measured.

However, considering a conservative assumption for the long term stability of the instrument, an absolute radiometric accuracy of $0.6 \mathrm{~dB}$ for StripMap Basic Products is ensured during the lifetime of TerraSAR-X. Thus, a whole SAR system at a distnace of more than $500 \mathrm{~km}$ could be successfully adjusted and calibrated with the accuracy of laboratory equipment.

\section{Conclusion}

The results of calibrating TerraSAR-X approve the accuracy calculated before launch and put the described strategy to calibrate efficiently a multiple mode SAR system like TerraSAR-X on a solid base. The key element of this strategy is an antenna model approach and TerraSAR-X is the first SAR satellite calibrated with this innovative method. It has been shown that all calibration systems are working very well. The stability and accuracy of the system and especially the radar instrument itself is of unprecedented quality. Tab. 1 lists the results of calibrating TerraSAR-X. All requirements and/or goals have been achieved even better than predicted. By this successful demonstration of an effective and exact calibration technique a new benchmark has been settled not only for calibrating complex SAR systems but in principle for future highly accurate spaceborne SAR sensors like TanDEM-X or Sentinel-1. The described calibration strategy has made an important contribution to achieve this aim.

\begin{tabular}{|c|c|c|}
\hline Calibration Procedure & Goal & Achieved \\
\hline \multicolumn{3}{|l|}{ Internal Calibration } \\
\hline Amplitude & $0.25 \mathrm{~dB}$ & $\pm 0.2 \mathrm{~dB}$ \\
\hline Phase & $1 \mathrm{deg}$ & $\pm 1 \mathrm{deg}$ \\
\hline \multicolumn{3}{|c|}{ T/R Module Characterization } \\
\hline Amplitude & $0.3 \mathrm{~dB}$ & $<0.2 \mathrm{~dB}$ \\
\hline Phase & $2.5 \mathrm{deg}$ & $<2$ deg \\
\hline \multicolumn{3}{|c|}{ Antenna Pointing Knowledge } \\
\hline Elevation & $0.015 \mathrm{deg}$ & $<0.006 \mathrm{deg}$ \\
\hline Azimuth & $0.002 \mathrm{deg}$ & $<0.002 \mathrm{deg}$ \\
\hline \multicolumn{3}{|c|}{ Pixel Localization Accuracy } \\
\hline Azimuth & $2 \mathrm{~m}$ & $0.5 \mathrm{~m}$ \\
\hline Range & $2 \mathrm{~m}$ & $0.3 \mathrm{~m}$ \\
\hline \multicolumn{3}{|c|}{ Antenna Model Verification } \\
\hline Elevation Pattern & $\pm 0.2 \mathrm{~dB}$ & $< \pm 0.2 \mathrm{~dB}$ \\
\hline Azimuth Pattern & $\pm 0.1 \mathrm{~dB}$ & $< \pm 0.1 \mathrm{~dB}$ \\
\hline $\begin{array}{l}\text { Beam-to-Beam Gain } \\
\text { Offset }\end{array}$ & $\pm 0.2 \mathrm{~dB}$ & $< \pm 0.2 \mathrm{~dB}$ \\
\hline \multicolumn{3}{|c|}{ Radiometric Accuracy (StripMap / ScanSAR) } \\
\hline Relative Accuracy & $0.68 \mathrm{~dB}$ & $0.3 \mathrm{~dB} / 0.4 \mathrm{~dB}$ \\
\hline Absolute Accuracy & $0.9 \mathrm{~dB}$ & $0.6 \mathrm{~dB} / 0.7 \mathrm{~dB}$ \\
\hline Radiometric Stability & $0.87 \mathrm{~dB}$ & $\pm 0.2 \mathrm{~dB} *$ \\
\hline
\end{tabular}

Table 1: Results of Calibrating TerraSAR-X

\section{References}

[1] M. Schwerdt, D. Hounam, J. L. Alvarez-Pérez, and T. Molkenthin, "The Calibration Concept of TerraSAR-X, a Multiple Mode High Resolution SAR," Canadian Journal of Remote Sensing, Vol. 31, No.1, Feb 2005 .

[2] B. Bräutigam, M. Schwerdt, M. Bachmann, and M. Stangl, "Individual T/R Module Characterisation of the TerraSAR-X Active Phased Array Antenna by Calibration Pulse Sequences with Orthogonal Codes," in 26th International Geoscience And Remote Sensing Symposium, Barcelona, Spain, 2007.

[3] B. Bräutigam, J. H. Gonzalez, M. Schwerdt, and M. Bachmann, "Radar Instrument Calibration of TerraSAR-X," in 7th European Conference on Synthetic Aperture Radar, Friedrichshafen, Germany, 2008.

[4] M. Schwerdt, B. Bräutigam, M. Bachmann, B. Döring, D. Schrank, and J. H. Gonzalez, "Final Results of the Efficient TerraSAR-X Calibration Method," in 2008 IEEE Radar Confrerence, Rome, Italy, 2008.

[5] M. Bachmann, M. Schwerdt, B. Bräutigam, and B. Döring, "Final results of the TerraSAR-X In-Orbit Antenna Model Verification," in 7th European Conference on Synthetic Aperture Radar, Friedrichshafen, Germany, 2008. 